Research Article

\title{
Gobernanza Colaborativa para la Innovación Pública y Social: El caso de Gipuzkoa, País Vasco
}

\section{Collaborative Governance for Public and Social Innovation: The case of Gipuzkoa, Basque Country}

\author{
Xabier Barandiaran \\ Departamento de Ciencias Sociales y Humanas, Universidad de Deusto, San Sebastián. \\ Correspondencia: xabier.barandiaran@deusto.es
}

\begin{abstract}
Resumen: La globalización y sus condiciones de desarrollo han puesto encima de la mesa una nueva agenda política caracterizada por la necesidad de abordar desafiantes retos sociales. Asimismo, la crisis de la democracia liberal ha evidenciado la necesidad de nuevos modelos para la gestión del espacio público y de las políticas públicas. Este artículo aborda el concepto de la Gobernanza Colaborativa como un mecanismo para estimular los procesos colectivos de deliberación a través del establecimiento de decisiones formales orientadas al consenso para identificar prioridades y generar líneas de actuación en los territorios. En este marco, el artículo presenta la experiencia práctica de Gobernanza Colaborativa "Etorkizuna Eraikiz", programa diseñado e implementado por la Diputación Foral de Gipuzkoa, explicitando los elementos estructurales que componen el programa y analizando el funcionamiento del modelo en el que se enmarca.
\end{abstract}

Palabras clave: gobernanza colaborativa; innovación pública; innovación social; capital social; Gipuzkoa; País Vasco.

\begin{abstract}
Globalization and its conditions of development have brought about a new political agenda characterized by the need to address social challenges. Similarly, the crisis of liberal democracy has highlighted the need for new models for the management of public space and public policies. This article addresses the concept of collaborative governance as a mechanism to stimulate collective processes of deliberation through the establishment of formal, consensus-oriented decisions to identify priorities and generate lines of action in the territories. Within this framework, the article presents the practical experience of collaborative governance, 'Etorkizuna Eraikiz', a program designed and implemented by the Provincial Council of Gipuzkoa, and explains the structural elements that comprise the program and analyzes the functioning of the model in which it is framed.
\end{abstract}

Keywords: collaborative governance; public innovation; social innovation; social capital; Gipuzkoa; Basque Country.

\section{Introducción}

Este artículo tiene como objetivo fundamental exponer los principios fundamentales en los que se basa el programa de Gobernanza Colaborativa "Etorkizuna Eraikiz" (Construyendo el 
futuro) de la Diputación Foral de Gipuzkoa, además de explicitar los elementos estructurales más relevantes que componen el programa y analizar el funcionamiento del modelo en el que se enmarca.

De esta manera, el desarrollo de este trabajo se divide en tres apartados. En primer lugar, profundiza en el contexto de la crisis de la democracia liberal; una crisis que se manifiesta en el debilitamiento de las estructuras políticas para dar respuesta a los retos económicos, sociales y políticos planteados y en la desafección de la ciudadanía respecto al sistema, las instituciones y los actores.

En segundo lugar, aborda la conceptualización de la gobernanza colaborativa como mecanismo para institucionalizar la construcción de la realidad política incorporando a la sociedad organizada y a la sociedad civil al sistema de deliberación pública; un mecanismo de gobernanza pública para hacer frente a la desafección política y responder, desde una visión sistémica, a las necesidades de los diversos ecosistemas que integran el diseño y la implementación de las políticas públicas.

En tercer lugar, presenta el programa Etorkizuna Eraikiz, una estrategia de Gobernanza Colaborativa puesta en marcha por la Diputación Foral de Gipuzkoa a través de la cual se ha institucionalizado un modelo integrado por procesos de deliberación compartida (Gipuzkoa Taldean), un laboratorio de anticipación y experimentación activa de políticas públicas (Gipuzkoa Lab) y un conjunto de Centros de Referencia que impulsan el desarrollo de la nueva agenda política en ámbitos estratégicos para el futuro del territorio.

\section{La globalización y la crisis de la democracia liberal}

En todo el mundo y también, en las sociedades occidentales, se están produciendo cambios económicos, sociales y políticos a una enorme velocidad. La razón fundamental que opera en esta transformación tiene que ver con dos grandes cuestiones. En primer lugar, con el desarrollo científico y tecnológico y su aplicación a los sistemas de generación de conocimiento y de producción. En segundo lugar, con el "modelo" de desarrollo del sistema capitalista de libre mercado que está, a su vez, íntimamente ligado al sistema de las democracias liberales.

El crecimiento económico impulsado por aquellos países con mayor capacidad para producir conocimiento, procesos y productos ha generado nuevas formas de pensar, sentir y actuar, alimentando un modelo de globalización. En este contexto, Ulrich Beck (1998) define la globalización como aquellos "procesos en virtud de los cuales los Estados nacionales soberanos se entremezclan e imbrican mediante actores transnacionales y sus respectivas probabilidades de poder, orientaciones, identidades y entramados varios" (Beck, 1998: 29). De acuerdo con Giddens (2007), la globalización es un concepto multidimensional que integra elementos políticos, tecnológicos, culturales y económicos que se ha visto fuertemente influenciado por los sistemas de comunicación (Giddens, 2007: 7). Implica necesariamente la integración de procesos que se producen a una escala mundial superando las escalas de vida que se originan en el contexto de los estados-nación o a escalas inferiores en otros períodos históricos configurando, por tanto, un nuevo espacio.

La configuración de este nuevo espacio involucra inevitablemente la apertura de un nuevo mundo (con sus condiciones de relación) y la modificación de los espacios de vida anteriores debido al influjo que ejerce el proceso de globalización. En este sentido, Ulrich Beck (1998) señala que la actual sociedad mundial está condicionada por un conjunto de relaciones sociales y de poder organizadas en un contexto no nacional-estatal, característica que posibilita la actuación por encima de las fronteras y que diluye la unidad del Estado, sociedad e individuo (Beck, 1998: 146). En esta misma línea, Simone (2016), en un interesante análisis sobre la frustración generada por las falsas expectativas de la democracia, subraya la evaporización del concepto frontera que ha producido la globalización (Simone, 2016: 163).

El proceso de globalización económica arrastra y condiciona al sistema político, provoca la generación de unas condiciones sociales determinadas en muchos países y, sobre todo, genera 
una cultura transnacional con contenidos de aquellos países que lideran los procesos globales y que influyen en el resto. Desde una posición crítica Beck (1998) habla de "globalismo", en el sentido de que el mercado mundial suplanta al quehacer político, imponiendo una ideología de mercado o del liberalismo, reduciendo el proceso de globalización fundamentalmente a una dimensión económica que aborda de manera lineal las dimensiones relacionadas con la ecológica, la cultura, la política y lo social (Beck, 1998: 27). En esta misma línea, Augusto de Venanzi (2002) concibe el término globalización como "el proceso de expansión desregulada del sistema de la gran corporación privada" (De Venanzi, 2002: 46). Este enfoque vincula no solo la dimensión económica a los procesos de globalización, sino que añade otras dimensiones que están fuertemente influenciadas por el marco en el que opera el sistema de la gran corporación privada. Estas dimensiones se relacionan con lo social, desde la perspectiva de la reconfiguración de la división social del trabajo; lo cultural, a través de la universalización de los valores occidentales; la política, a partir de la integración de un sistema normativo de orden supranacional; y la ecológica, a través de la apropiación de la naturaleza y el deterioro del medio ambiente (De Venanzi, 2002: 51).

Thomas Piketty (2021) advierte sobre las diferencias estructurales entre países que tienen capacidad para acceder al área global y los que no tienen esa capacidad. Otros autores subrayan también las diferencias estructurales que se están produciendo entre las grandes metrópolis y las pequeñas ciudades y regiones del interior de los países occidentales. A este respecto, Guilluy (2019) señala, por ejemplo, la desaparición de la clase media y el abandono de las clases altas respecto a los más desfavorecidos. El autor expone que la sociedad abierta y globalizada en la que vivimos hoy, se ha alejado de las necesidades del pueblo en general y, como consecuencia, aquellos con mayor capacidad han replegado a sus fortalezas, sus empleos y sus riquezas (Guilluy, 2019: 96). Por tanto, la desigualdad y las condiciones sociales, políticas y económicas en las que se desarrolla el modelo actual de globalización constituyen el principal factor de la crisis de las democracias liberales.

En este contexto, hay que destacar tres cuestiones centrales a tener en cuenta a la hora de analizar la crisis de la democracia liberal. Primero, las consecuencias de la superación del marco del estado-nación. Segundo, la emergencia de las sociedades individualistas y de consumo que transforman la construcción social de la realidad política. Tercero, las nuevas formas de producción social de la realidad a través de las nuevas tecnologías de la información y la comunicación.

La transformación de la realidad social y la generación de condiciones públicas democráticas dependen en gran medida de actores económicos, políticos y sociales que no interactúan bajo las condiciones del estado-nación; esta realidad implica una menor capacidad de transformación de las estructuras políticas estatales pero también muestra la emergencia de escenarios políticos, sociales y económicos desequilibrados propios de un contexto desregulado donde priman las condiciones de desarrollo impuestas por aquellos actores que tienen más capacidad de influencia.

Por su parte, la atomización de las estructuras de socialización política junto a una sociedad individualista orientada al consumo genera interacciones sociales fragmentadas que dificultan la construcción de narrativas sociales compartidas. Hoy, en las sociedades occidentales, la ciudadanía tiende cada vez más a comprender el hecho político como un fenómeno ajeno que es consumido, en muchos casos, como producto mediático en respuesta a los estímulos marcados por la agenda electoral. En cierta medida los estados tienen menos capacidad de influir y la ciudadanía actúa, cada vez más, como un cliente que espera su servicio y no como un ciudadano sujeto a una comunidad política con derechos y obligaciones. El cambio de posición y el rol de los actores en el sistema político (ciudadanía, partidos e instituciones) es motivado por un cambio económico y social generador de una realidad política distinta.

En los países occidentales, por ejemplo, las encuestas de opinión pública nos muestran una ciudadanía con poco interés por la política y un conocimiento escaso de la realidad política, de la comunidad política, del espacio de opinión pública, de los elementos que configuran el sistema 
político e institucional. Los actores políticos compiten por la adhesión política y los contenidos de esta competencia están muy condicionados por dos grandes elementos. En primer lugar, por la forma en la que los ciudadanos consumen el hecho político (escenarios de producción y consumo audiovisuales) y, en segundo lugar, por la relación estructural que la ciudadanía mantiene con la política (enorme distancia, relación superficial, etc.). El debate y la relación política entre los actores políticos está centrado en la disputa de titulares que puedan ser objeto de consumo. Esta lógica de relación condiciona de manera significativa a los actores políticos también en la gestión política de la administración y de las políticas públicas. Así, la transformación de las condiciones de legitimación de la ciudadanía respecto a los actores políticos está transformando la función y el sentido político de su actividad pública en el sistema institucional (el tiempo dedicado a la comunicación política y a la construcción de escenarios de comunicación es un buen ejemplo de esta realidad).

En el despliegue de este contexto, cabe señalar una última cuestión. La globalización y sus condiciones de desarrollo han puesto encima de la mesa una nueva agenda política caracterizada por la necesidad de abordar retos sociales como el cambio climático, la gestión de la diversidad, la lucha contra las desigualdades, la seguridad de los espacios públicos, las nuevas condiciones individuales y colectivas de vida producidas por la digitalización, etc. Nos enfrentamos a una nueva agenda política $\mathrm{y}$, además, a condiciones de desarrollo condicionadas por la incertidumbre, la disrupción y la falta de estabilidad. La pandemia ha sido una sorpresa para todas las sociedades; responsables públicos, diversos sectores económicos y sociales y, en general, el conjunto de la ciudadanía se ha visto incorporado de forma violenta a un nuevo escenario caracterizado por el miedo, la inseguridad y la incertidumbre. El virus está siendo un factor de aceleración de las tendencias económicas, sociales y políticas que se venían produciendo en el contexto de la globalización y, sobre todo, debido a su fuerte impacto se ha constituido en una fuerza resocializadora de primer orden. La pandemia está afectando nuestra condición como seres humanos y perturbando las diversas realidades de la acción colectiva.

Por tanto, una de las grandes cuestiones que se ha puesto encima de la mesa es cómo hacer frente a esta nueva situación y, en este sentido, la Gobernanza Colaborativa puede contribuir a un cierto restablecimiento de las condiciones públicas para la construcción de la democracia en el actual contexto global.

\section{La Gobernanza Colaborativa como respuesta a la crisis de la democracia}

La crisis de la democracia liberal remite a la búsqueda de nuevos modelos para la gestión del espacio público y de las políticas públicas. Formas de acción y modelos que reestablezcan la democracia y que incorporen a la sociedad en el espacio deliberativo.

El concepto de Gobernanza emerge en los países occidentales en los años 90 como un paradigma que da cuenta del cambio de rol del Estado y su forma de gobernar la relación con los actores de la sociedad organizada, la sociedad civil y el mercado. Partiendo de la reflexión de que los gobiernos dependen en gran medida de la cooperación y de los recursos de la sociedad en general para dar respuesta a los asuntos públicos (Kenis \& Schneider, 1991: 36), el concepto de Gobernanza transita de un modelo burocrático y jerarquizado a un modelo más cooperativo en el que se establece una complementariedad entre el sector público, el sector privado, los grupos y ciudadanos que constituyen la sociedad civil (Conejero, 2016). El concepto ha tenido diversas aproximaciones condicionadas por la disciplina desde la que se ha utilizado el termino y el rol que se atribuye al Estado; esto da lugar a definiciones centradas en el Estado o en actores que interactúan en el sistema (Whittingham, 2010).

Tal y como señala Antonio Natera (2005), el valor de la gobernanza reside fundamentalmente en la capacidad de establecer un marco para entender el análisis de la relación del Estado con la sociedad. La Gobernanza alude a una nueva forma de establecer la dirección política y la gestión de las políticas públicas. Una nueva forma de comunicación entre los responsables políticos y los actores organizados o individuales de la sociedad civil. Y aunque se 
utilicen distintas denominaciones para incorporar a la sociedad a la deliberación pública, como "Ciudadanía Colaborativa" (Smith, 2010), "Compromiso Comunitario" (Head, 2008), "Gestión Pública enfocada al Ciudadano" (Cooper, 2005); de manera particular, la Gobernanza Colaborativa está orientada a compartir decisiones públicas con la sociedad.

Desde esta visión, el trabajo aquí presentado, no pretende desplegar el estado del arte de la conceptualización teórica sobre la Gobernanza Colaborativa, pero intenta exponer las referencias fundamentales en las que se enmarca la edificación de la visión de gobernanza en el contexto del programa estratégico Etorkizuna Eraikiz, el cual será presentado posteriormente.

En este contexto, vale la pena destacar que hablar de la Gobernanza Colaborativa es hablar del establecimiento de las condiciones estructurales y culturales que garantizan la calidad democrática para la deliberación y para la acción de los actores públicos, privados y sociales que interactúan en un contexto dado de políticas públicas.

De manera concreta, Sirianni (2010) define la Gobernanza Colaborativa como el contexto en el cual los administradores públicos interactúan con el conjunto de la sociedad para estimular procesos colectivos de deliberación que permiten identificar prioridades y generar líneas de actuación, frente a diversas situaciones, que tengan como base las fortalezas existentes en las comunidades y en los individuos.

La perspectiva polícéntrica en la que se asienta la Gobernanza Colaborativa, aborda la propia gobernanza como un proceso que vincula al Estado o a las estructuras públicas, a la sociedad civil (en sus diversas formas de manifestación en cuanto al interés público) y a actores privados (Whittingham, 2010). En este sentido, es importante resaltar que la razón por la cual interesa esta perspectiva es porque, tal y como lo expone Aguilar (2020), en el contexto de los cambios que se han producido en la política a raíz de la globalización, el debate se encuentra focalizado en la insuficiencia de los gobiernos democráticos para dirigir e influir a través de las políticas públicas en sus respectivas sociedades. De ahí, la plena identificación con la afirmación que hace Aguilar (2020), en el sentido de que hoy más que nunca se demanda una nueva forma de gobernar, de orientar y coordinar a la sociedad. El gobierno por sí solo no puede definir y desarrollar la agenda de la sociedad y mucho menos dar respuesta a los numerosos asuntos públicos que inquietan a la sociedad contemporánea (Aguilar, 2020: 72). Además, en la actual realidad global, las situaciones y los asuntos de interés colectivo no pueden ser conocidos, abordados o dirigidos por un solo actor o una sola estructura de acción (Aguilar, 2008: 24). De manera semejante, Sonia Ospina (2006) advierte que lo público ya no es responsabilidad exclusiva del Estado y pasa a ser más bien un espacio de interacción entre diversos actores.

El actual modelo de desarrollo provocado por la globalización ha limitado significativamente la capacidad de transformación de las estructuras políticas. La construcción de una comunidad política está condicionada por múltiples factores y a esta complejidad se añade el hecho de que la lógica de la actual sociedad está inmersa bajo los patrones de consumo e individualismo. En las sociedades actuales, el nivel de solidez y cohesión de la comunidad ha disminuido y las condiciones para el desarrollo y la legitimación del espacio público plantea enormes retos desde el punto de vista democrático y de eficacia. De manera que, si proliferan los factores, estructuras y actores de la transformación de la comunidad política, el entramado de políticas públicas que requiere el ejercicio de la gobernanza del espacio público debe ser inevitablemente redefinido y empoderado para ganar legitimación democrática y poder de transformación. En este aspecto, existe un consenso bastante generalizado sobre la idea de la insuficiencia en la acción de gobierno de las administraciones públicas, particularmente, en autores relevantes que han trabajado la Gobernanza Colaborativa desde el punto de vista de redes (Kooiman, 2000; Sorensen \& Torfing, 2009; López \& Lavié, 2010).

En tal sentido, el enfoque de este trabajo se desarrolla desde la perspectiva de redes y desde una visión en la que la administración pública tiene un papel de liderazgo fundamental (Peters \& Pierre, 2005). Desde esta perspectiva, es necesario destacar que Rhodes (2007) define la gobernanza como el proceso de gobernar con y a través de redes. Asimismo, este enfoque 
fundamento en el impulso de dinámicas de interacción y trabajo en red ha sido subrayado por otros autores como Ilyin (2013) y Atkinson y Coleman (1992).

La perspectiva introducida por Rhodes (1999), plantea la interdependencia entre las organizaciones, la interacción para el intercambio de recursos y la negociación para compartir objetivos y construir interacciones de confianza a partir de unas reglas de juego pactadas entre los actores vinculados. Por tanto, es desde este enfoque de la gobernanza en red que emerge también el concepto de la Gobernanza Colaborativa.

Ansell y Gash (2008), al referirse al concepto de Gobernanza Colaborativa, subrayan la importancia de la interacción entre actores públicos y actores no estatales a través del establecimiento de un proceso colectivo de decisiones formales, orientado al consenso y la deliberación con el objetivo de implementar las políticas públicas o desarrollar programas públicos. Este modelo de gobernanza vincula a múltiples actores con organismos públicos a través de espacios comunes que potencian la participación y el consenso en la toma de decisiones (Ansell \& Gash, 2008). Tal y como señalan estos autores, este proceso implica un impulso de las instituciones públicas, la inclusión en el proceso de actores no estatales y que el proceso no sea exclusivamente consultivo sino decisorio, que sea un proceso deliberativo formalmente establecido, en el que se logren consensos y el foco de la deliberación esté centrado en las políticas públicas.

De otro lado, en la medida en que la definición de Ansell y Gash (2008) incorpora procesos formalmente instituidos, Emerson, Nabatchi y Balogh (2011) amplían el concepto de Gobernanza Colaborativa definiéndolo como "aquellos procesos y estructuras de decisión y gestión política y pública que conectan constructivamente a personas entre los diferentes ámbitos y departamentos de las agencias públicas, niveles de gobierno, y/o esferas públicas, privadas o cívicas para llevar a cabo o dar respuesta a una solución difícilmente alcanzable por otros cauces" (Emerson, Nabatchi \& Balogh, 2011: 2). De la misma forma, Arellano, Sánchez y Retana (2014) vienen a definir el concepto de Gobernanza Democrática destacándola como "un proceso en el que el gobierno, las organizaciones privadas y la sociedad civil interactúan para decidir, coordinar y llevar a cabo la dirección y gobierno de su comunidad" (Arellano, Sánchez \& Retana, 2014: 121). Calame y Talmant (1997) añaden, además, la capacidad de la Gobernanza Colaborativa para conceptualizar nuevos sistemas de representación y de instituir nuevas formas de acción en un proceso de adaptación.

En ese marco, la Gobernanza Colaborativa se ha convertido en un término común en la literatura sobre la administración pública (Ansell \& Gash, 2008; O’Leary \& Bingham, 2009; Cooper, Bryer \& Meek, 2006; Fung, 2006), siendo un concepto destacado por incrementar la capacidad de la administración pública a raíz del debilitamiento derivado de las transformaciones económicas, políticas y sociales del mundo actual. Como contribución a los marcos teóricos desarrollados, este trabajo define la Gobernanza Colaborativa cómo el proceso de deliberación y acción compartida que vincula a las instituciones públicas, la sociedad organizada y la sociedad civil en orden a fortalecer, a través de la generación de Capital Social, una nueva cultura política en el ecosistema de las políticas públicas (en su diseño y procesos de ejecución) en el contexto de un espacio público compartido.

Retomando el planteamiento realizado por Aguilar (2020), vale la pena destacar las características distintivas de la Gobernanza en red que este autor presenta. En primer lugar, destaca la naturaleza plural de los actores participantes en el ecosistema de la red, como segundo elemento, la interdependencia de los actores, el establecimiento de las formas de colaboración y la corresponsabilidad que esto implica. En tercer lugar, se enfatiza en el compromiso de compartir recursos y riesgos para el logro de los fines propuestos y las normas que establecerán las condiciones de relación en el proceso de deliberación y de ejecución.

De modo similar, Subirats (2010) señala también algunas premisas básicas que permiten identificar el concepto de Gobernanza, haciendo énfasis en el reconocimiento e incorporación de la complejidad como un elemento consustancial al hecho político, la participación de los actores 
en el marco de redes plurales y, sobre todo, la aceptación de una nueva posición en los procesos de gobierno. Esta línea es seguida también por Cantó (2012) quien reflexiona sobre la pérdida de poder de las administraciones, en la medida en que no solamente el gobierno administra y ejecuta sino también lo hacen las redes auto-organizadas y el mercado. Insiste, asimismo, en la importancia de la participación de la sociedad civil y el sector privado en la toma de decisiones, la co-creación, la corresponsabilidad y el incremento del nivel de eficacia y de la legitimidad democrática en los procesos de Gobernanza Colaborativa.

Por todo lo anterior, partiendo del marco general de lo que se entiende por Gobernanza Colaborativa es necesario destacar tres implicaciones fundamentales que derivan de este concepto. La primera implicación está relacionada con la redefinición del espacio deliberativo a través de la incorporación de nuevos actores y la transformación de la lógica del poder en un poder compartido. La segunda, tiene que ver con la necesidad de generar capital social para activar una nueva cultura política que permita dar respuesta a una estructuración institucional en términos de Gobernanza Colaborativa. Finalmente, la tercera implicación corresponde a la generación de condiciones para la innovación social desde el punto de vista de la co-creación y la experimentación activa con el objeto de dar respuesta a las múltiples necesidades que se plantean en un contexto de complejidad e incertidumbre.

\subsection{Redefinición del espacio deliberativo y la distribución del poder}

La incorporación de diversos actores en la deliberación pública nos lleva de manera inevitable a reflexionar sobre cómo se configura ese nuevo espacio en términos democráticos. ¿A quién representan los distintos stakeholders que participan en el proceso de deliberación? En este sentido, Aguilar (2020) señala que el rol directivo del gobierno no desaparece en la medida en que es la autoridad legítima de la asociación política y garante de preservar la naturaleza pública del proceso respetando los valores y principios democráticos fundamentales. Ahora bien, el rol político se ejerce a través de la coordinación y no a través de la imposición jerárquica. La visión presentada por Aguilar (2020), mantiene la preeminencia de la estructura pública, pero introduce el concepto post-gubernamental de la gobernanza pública para redefinir el nuevo espacio de las políticas. De manera que, la incorporación de los diversos actores sociales, económicos, políticos de diversa naturaleza implica un factor de redefinición del espacio público, en cuanto que suma al espacio público, derivado de la estructura pública, otros espacios que no derivan de la estructura pública pero que responden al interés general y desarrollan una función social clara. El Estado y la administración pública no absolutizan ni agotan toda la realidad construida en el espacio público. Esta cuestión plantea evidentes problemas que es necesario abordar a través del diálogo y la negociación. Dichas problemáticas se enmarcan en cuestiones como la diversidad y los frecuentes antagonismos en la conceptualización y significación de los objetos políticos, las visiones contrapuestas, los desequilibrios en las capacidades, las habilidades y las oportunidades, las desiguales condiciones de poder y la autoridad como punto de partida, la dificultad de desarrollo de una deliberación compartida pero también de los procesos de ejecución, etc. Sin embargo, como afirma Aguilar (2020), estos problemas no pueden llevarnos a aceptar la tesis de que la incorporación de actores privados y sociales distorsiona el objetivo público de gobernar.

En torno a la redefinición del espacio deliberativo que configura la Gobernanza Colaborativa se ha planteado un importante debate académico sobre la significación del poder, sobre su distribución y sobre cómo afectan las asimetrías de poder o los desequilibrios de este al nivel de efectividad del desarrollo de la Gobernanza Colaborativa. Ansell y Gash (2008) advierten sobre el riesgo de manipulación del proceso deliberativo por parte de los actores más poderosos.

Por tanto, ¿Es suficiente la institucionalización de una interacción formal y estable entre actores públicos, sociales y privados para poder hablar de Gobernanza Colaborativa?; ¿Es suficiente seguir formalmente un proceso de Gobernanza Colaborativa, por ejemplo, a través de un proceso de co-creación y acción compartida entre los stakeholders, para hablar de Gobernanza Colaborativa? La Gobernanza Colaborativa exige tener en cuenta la articulación de las 
condiciones de la distribución del poder y una cultura política colaborativa interiorizada por parte de los actores que participan en los procesos para lograr establecer un mecanismo efectivo de gobernanza basado en la colaboración. En consecuencia, Run, Huiting y Oszlak (2018) consideran necesario el establecimiento de un marco que posibilite el entendimiento de las relaciones de poder que se producen en un marco de Gobernanza Colaborativa. De igual forma, Hardy y Philips (1998) apuntan a tres elementos clave: la autoridad formal, el control de los recursos críticos y la legitimidad discursiva. Lo cierto es que además de estos factores, existen otros como el contexto social e institucional que condicionan esas relaciones de poder.

Run, Huiting y Oszlak (2018) consideran fundamental evaluar el nivel de efectividad de los procesos de Gobernanza Colaborativa a través de distintas formas de medición como el grado de colaboración, los resultados de la acción o la medición de la satisfacción de los stakeholders. Diversos autores apuntan a los beneficios de compartir el poder, pero Run, Huiting y Oszlak (2018) consideran que todavía falta un marco contingente adecuado para analizar la relación entre compartir el poder y la afectividad en la colaboración. Esta es una cuestión central en la Gobernanza Colaborativa, y para la que estos autores plantean seis elementos que permiten trabajar en dicha relación (Run, Huiting \& Oszlak, 2018: 61-74):

1) Cuanto más fuerte es el contexto institucional en cultivar la colaboración más beneficioso resulta compartir poder para una Gobernanza Colaborativa efectiva.

2) Cuanto menos exigente es la misión cumplida por la Gobernanza Colaborativa, más beneficioso resulta compartir poder para la efectividad de la Gobernanza Colaborativa.

3) Cuanto más voluntaria es el tipo de Gobernanza Colaborativa, más beneficioso resulta compartir poder para una mayor efectividad de la gobernanza.

4) Cuanto menor es la experiencia de compartir poder de los participantes, menos beneficioso será compartir poder a efectos de la efectividad de la Gobernanza Colaborativa.

5) Cuando más ampliamente difundidas las fuentes de poder, mayor será el beneficio de compartir poder para la efectividad de la Gobernanza Colaborativa.

6) Cuanto más aceptable es el cálculo de costo-beneficio para los participantes, más beneficioso será compartir poder para la efectividad de la gobernanza.

\subsection{La generación de Capital Social}

El Capital Social está íntimamente ligado a los procesos de Gobernanza Colaborativa en la medida en que la incorporación de actores privados, sociales o de cualquier otro tipo al sistema deliberativo supone la creación de nuevas redes y también la posibilidad de generar normas y valores que redefinen nuevas interacciones sociales de calidad que posibilitan sistemas de cocreación y de acción de las políticas públicas. Para Robert Putnam (1993) el Capital Social se define como un conjunto de interacciones sociales institucionalizadas o formalizadas en distinto grado (que incluyen valores y normas sociales de reciprocidad y confianza) que influyen en el desarrollo comunitario. Putnam (1993) presta especial atención a la sociedad civil diferenciando al mercado y al Estado. El Capital Social favorece la cooperación y la interacción se constituye en un recurso porque la estructura de esa interacción obliga a la reciprocidad.

El Capital Social hace referencia a las conexiones entre personas, redes sociales, normas de reciprocidad y la confianza que se construye en esas relaciones (Putnam, 2000). En la medida en que determinados contextos interiorizan actitudes y valores que provocan la coordinación y cooperación de los actores sociales, aumenta la capacidad de generar redes o procesos de institucionalización formal e informal que desemboca en la capacidad de un entorno para dar respuesta a las necesidades que se plantean. Al mismo tiempo, en la medida de la existencia de un entorno articulado en redes se va generando un proceso de socialización de los valores cooperativos en un entorno. Es una espiral que se va autoalimentando en una dirección positiva o negativa. 
Desde otra aproximación, para Coleman (1988) el Capital Social puede tomar muchas formas, entre las que están las obligaciones que hay dentro de un grupo, las normas y las sanciones. En el caso de Bourdieu (1983), el concepto de Capital Social subraya las relaciones sociales que incrementan la capacidad de un actor para promover sus intereses. De nuevo aparece la idea de que el Capital Social permite al individuo específico ir más allá de sí mismo/a en la medida en que le facilita la colaboración y el compromiso, así como el flujo de información; mediante un sentido de pertenencia y obligación social, puede ejercer influencia sobre los demás (Saffer, 2016). La aceptación de la noción del Capital Social como concepto implica entender que las relaciones son fuente de desarrollo.

Bourdieu (1983), Coleman (1988, 1990), Fukuyama (1995), Putnam (2000) y otros científicos sociales subrayan que el nivel de confianza interpersonal, el compromiso cívico y la capacidad organizativa en una comunidad son factores de desarrollo. Estableciendo una vinculación clara entre Capital Social y Gobernanza. En el mismo sentido, Whittingham (2010) considera que un grupo o comunidad con una alta capacidad para actuar como un sistema coherente y cohesionado favorecerá las relaciones de gobernanza.

\subsection{Gobernanza e Innovación Social}

El tercer concepto clave que es necesario vincular a los sistemas de Gobernanza Colaborativa es el de la Innovación Social. Planteamos la necesidad de una Gobernanza Colaborativa como una alternativa a la crisis de la democracia liberal, para incorporar a la sociedad a la deliberación, para desarrollar nuevos espacios de creación, deliberación y acción; es decir, necesitamos una nueva gobernanza para formular nuevas preguntas, buscar nuevas respuestas para dar satisfacción a las nuevas demandas. Gobernanza Colaborativa para la Innovación Social. Es necesario superar la visión jerarquizada y funcionalista de la administración pública para dar paso a un modo de gobernanza en el que fluya la colaboración, la creatividad y la innovación social en red para buscar nuevas respuestas a las necesidades planteadas.

Tal y como señala Conejero (2016), la interacción entre actores involucrados en los procesos de Innovación Social exige repensar el modelo de gobernanza "los valores de autoridad y jerarquía que tradicionalmente han presidido las relaciones del gobierno con la sociedad deben dar paso, en un modelo de innovación social, a los principios de colaboración y cooperación horizontal entre actores" (Conejero, 2016: 17). Siguiendo esta misma dirección, y en línea con la tesis central de este artículo, Conejero (2016) considera que la crisis de legitimidad sólo puede ser superada con nuevos mecanismos de participación y de deliberación. Anshell y Gash (2008) afirman que el modelo de gobernanza, entendido como organización de la acción colectiva por medio de la institucionalización formal e informal, es uno de los elementos nucleares de los procesos de Innovación Social, porque afecta a las relaciones sociales y a la satisfacción de las necesidades básicas que se quedan al descubierto.

De otro lado, en un interesante trabajo Francisco Longo (2020) defiende que la administración pública tiene dos grandes retos: progresar (mejorar las políticas públicas, atender las exigencias de la ciudadanía cada vez más acostumbrada a funcionar en interacciones de mercado, tratar de ganar enormes ganancias de eficiencia y la activación del crecimiento económico y la prosperidad) y proteger (de la capacidad de opacidad del sistema financiero internacional, de las consecuencias de fuertes concentraciones empresariales a nivel global, del desempleo provocado por el avance tecnológico, de la vulnerabilidad tecnológica, de las desigualdades que se están generando en la sociedad). Sin embargo, el paradigma burocrático tradicional de la administración pública no puede abordar esos retos porque supone la existencia de contextos estables y desarrollos lineales, la existencia de un conocimiento de la materia a regular y se basa en el alejamiento de los supervisados como requisito de la aplicación impersonal de la norma (Longo, 2020).

Según Longo (2020), el Estado está obligado a superar su visión tradicional e innovar a través de la exploración y de la experimentación. El autor apuesta por la gobernanza exploratoria para 
afrontar la agenda de innovación que debe abordar la administración pública en un contexto de complejidad e incertidumbre. Longo (2020) señala que está gobernanza exploratoria debería de incorporar algunas características como poner el foco en la estrategia para liderar el cambio, estimular la inteligencia concentrando el mejor talento de la sociedad siendo la administración una red de núcleos de conocimiento más que un proceso vertical, promover la heterogeneidad y la descentralización para conciliar una diversidad creciente, garantizar el rigor en la evaluación de los procesos de cambio, fomentar la apertura y la conectividad.

\section{El caso Etorkizuna Eraikiz: Un modelo de Gobernanza Colaborativa}

Etorkizuna Eraikiz es una estrategia de la Diputación Foral de Gipuzkoa para afrontar la nueva agenda política y los retos estratégicos más relevantes del territorio a través de la Gobernanza Colaborativa.

Desde una visión abierta y participativa el objetivo es desarrollar las políticas públicas de manera compartida con la sociedad. Etorkizuna Eraikiz es también una forma de implicar a los ciudadanos. Una invitación a diseñar conjuntamente el futuro partiendo de la premisa de que mañana empieza hoy. Esta estrategia obliga a los políticos a reaprender y a abandonar la teoría y la burocracia para aplicar soluciones reales en escenarios reales. Obliga a gestionar de otra manera, con un nuevo modelo de gobernanza abierta y colaborativa que implique a organizaciones, empresas, asociaciones, universidades a trabajar juntos en pro de un futuro más igualitario para todos y todas. Se trata de la configuración de un modelo que escucha la voz de cada ciudadano y que despliega las estructuras necesarias para interactuar con él. En definitiva, un modelo que trabaja para encontrar nuevas respuestas, partiendo de los valores guipuzcoanos de cooperación y trabajo en equipo que identifican al territorio y le han permitido llegar a lo que es hoy. La Diputación Foral de Gipuzkoa define esta estrategia de la siguiente manera:

"Etorkizuna Eraikiz es un modelo, una forma peculiar y específica de entender, aplicar y representar la gobernanza abierta y colaborativa, una forma de "hacer política". El propósito de Etorkizuna Eraikiz es detectar colectivamente los retos futuros del territorio de Gipuzkoa, disenar la manera de afrontarlos, experimentar posibles respuestas en entornos reales con la colaboración de distintos agentes y aplicar los resultados al ecosistema de las políticas publicas de la Diputación Foral de Gipuzkoa" (Diputación Foral de Gipuzkoa, 2019).

Etorkizuna Eraikiz nace de la idea de que el modo con el que se haga frente a la magnitud de los retos demográficos, económicos, sociales y medioambientales que la sociedad tiene que afrontar en el futuro es el que sentará las bases del modelo social y económico a medio y largo plazo de Gipuzkoa.

\subsection{Los Principios de Etorkizuna Eraikiz}

Etorkizuna Eraikiz se asienta sobre cuatro principios que dan sentido a todo su desarrollo posterior:

1) El liderazgo institucional: El programa es promovido y liderado por la Diputación Foral de Gipuzkoa que actúa como el agente que lo propone y lo financia, además de participar activamente en su desarrollo. La Diputación Foral de Gipuzkoa manifiesta su compromiso con el programa no solamente facilitando la articulación e interacción entre los distintos agentes, sino comprometiéndose a que los diversos procesos deliberativos estén vinculados al desarrollo de las políticas públicas de la institución. El compromiso institucional se expresa, no solo en la vocación y el ejercicio de liderazgo, sino también en la creencia en la capacidad transformacional del programa y en la apuesta por su sostenibilidad a medio y largo plazo. Así los emprendedores institucionales (políticos) deben favorecer el cambio sistémico para lo cual es necesario crear las estructuras y las plataformas que permitan redefinir las relaciones entre las instituciones y la sociedad. Es decir, impulsar la transformación del sistema institucional hacia nuevos modelos de gobernanza. 
2) Coherencia con el Modelo Gipuzkoa: El programa se apoya en aquellas características del territorio que contribuyen a favorecer su desarrollo. Gipuzkoa es un territorio compuesto por comarcas muy dinámicas y avanzadas en lo que se refiere a sus recursos e infraestructuras socioeconómicas, con un tejido asociativo denso. Es una muestra de la capacidad y la cultura de la participación y el intercambio presente en el territorio. De igual forma, es un territorio con mucha iniciativa social. Está muy desarrollada en el territorio y una de sus manifestaciones históricas más relevantes es el movimiento cooperativo. La iniciativa social sigue hoy día expresándose en los ámbitos culturales, sociales o económicos; un territorio en el que se reconocen actitudes y experiencias bastantes extendidas de colaboración e interacción ciudadana, de capacidad de afrontar, resolver retos de manera compartida y elevado nivel de Capital Social.

3) Contextos abiertos de aprendizaje y experimentación: La idea de contexto abierto sugiere la intervención de un conjunto amplio de agentes y agencias que dan sentido a los procesos de cooperación y dan también forma a los resultados que puedan obtenerse. Esta concepción abierta requiere aprendizaje y capacidad de orientar, distribuir y expandir las experiencias colectivas. Por su propia naturaleza la colaboración público-privada implica interacción entre distintas culturas y experiencias organizativas y deviene, por tanto, en un proceso de aprendizaje que debe superar barreras y convertir en rutina la cooperación entre diferentes.

4) Generación de democracia, confianza y valor público: El Programa Etorkizuna Eraikiz, en tanto generador de democracia, confianza y valor público debe necesariamente situarse por encima del partidismo político. Debe plantear un modelo de gestión administrativa y gobernanza política capaz no solo de estimular sino de estabilizar una nueva relación de cooperación y cercanía entre la ciudadanía, las agencias sociales intermedias y las instituciones públicas que fortalezca la democracia y la confianza pública; la producción de resultados capaces de generar bienes públicos (tangibles e intangibles) y, en tal sentido, valor público y, finalmente, generar la capacidad para transformar las políticas públicas tanto en su concepción, como en su gestación y desarrollo.

\subsection{La estructura de Etorkizuna Eraikiz}

Etorkizuna Eraikiz está edificado sobre tres espacios fundamentales: Gipuzkoa Taldean, Gipuzkoa Lab y los Centros de Referencia.

1) Gipuzkoa Taldean: Gipuzkoa Taldean es el espacio para la escucha activa, para la deliberación a través de la metodología de investigación-acción con el objetivo de incidir en el ecosistema de las políticas públicas, tanto desde el punto de vista de los contenidos como desde el punto de vista de las formas y metodologías del desarrollo de la acción. Está configurado por varios programas, pero uno de los más significativos es Etorkizuna Eraikiz Think Tank. La misión de este programa es la de co-generar, a través de la Gobernanza Colaborativa, conocimiento transferible y aplicable que incida en una nueva agenda y cultura política que modernicen el ecosistema (actores, contenidos y procesos) de las políticas públicas de la Diputación Foral de Gipuzkoa. La metodología utilizada para el desarrollo co-generativo basado en la Gobernanza Colaborativa es el de la investigación-acción. La investigación-acción es una investigación orientada a la resolución de algún problema o reto acordado entre los participantes en un proceso. En este proceso confluyen el conocimiento metodológico, el conocimiento basado en la experiencia y el conocimiento teórico. El Instituto Vasco de Competitividad-Orkestra es la organización encargada de liderar la labor de facilitación y desarrollo metodológico de este Think Tank, representado en la Figura 1. En este marco, Etorkizuna Eraikiz Think Tank opera bajo el esquema metodológico referencial que proponen Coghlan y Branick (2001). 
El Think Tank se articula a través de cuatro grupos de reflexión-acción en el que participan, junto a responsables políticos y técnicos de la Diputación Foral de Gipuzkoa, actores de la sociedad organizada en diversas materias: El Trabajo del futuro, la Recuperación verde, Los futuros del estado del bienestar y la Nueva cultura política.

Figura 1. Esquema metodológico-referencial Think Tank.

\section{INVESTIGACIÓN ACCIÓN}

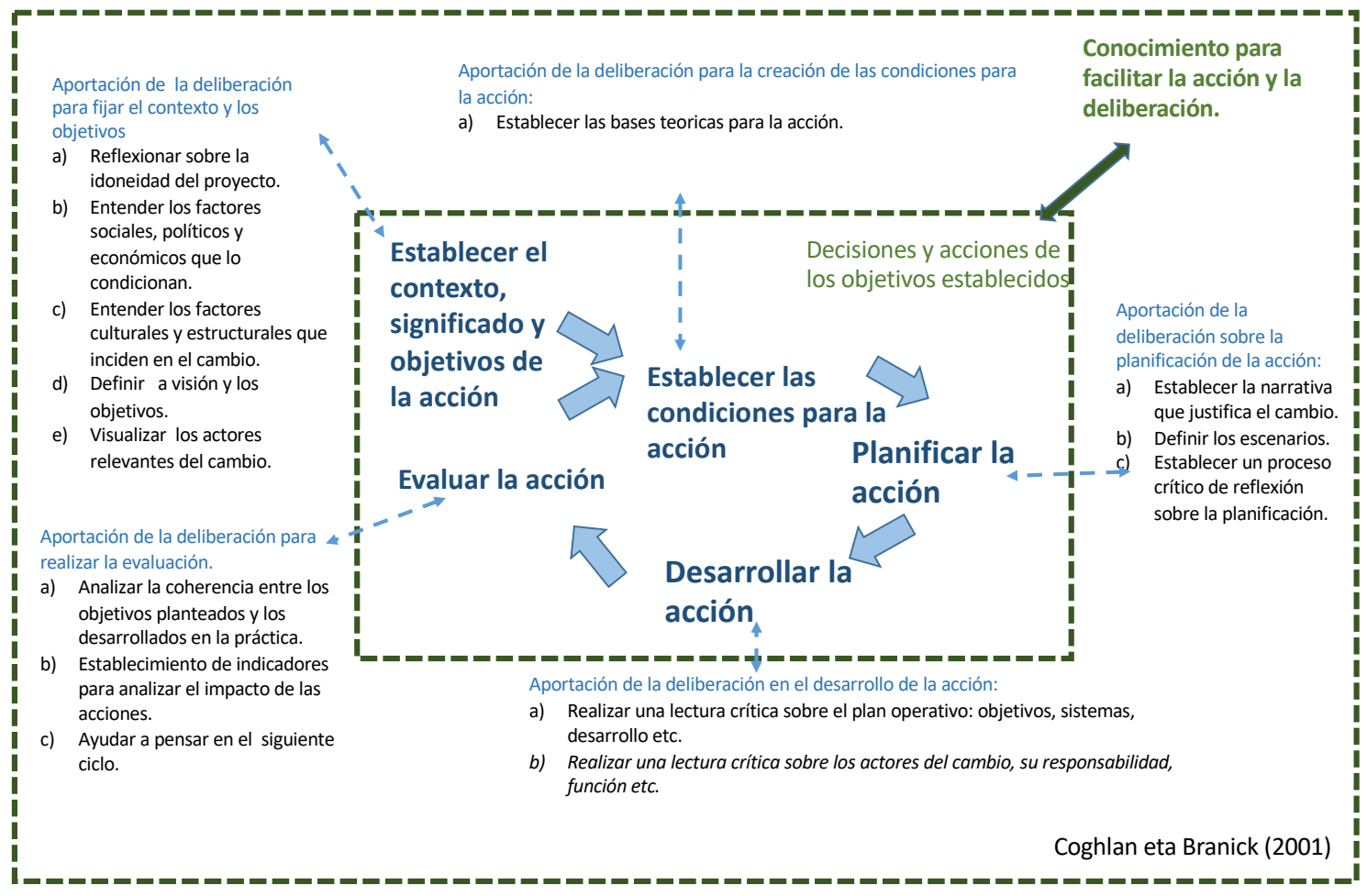

Fuente: Instituto Vasco de Competitividad-Orkestra a partir de Coghlan y Branick (2001).

El Think Tank completa su actividad con diversas actividades orientadas a la difusión, como la investigación en materias que tienen que ver con la agenda política.

De otro lado, el espacio de trabajo de Gipuzkoa Taldean desarrolla otras actividades que vienen a fortalecer el proceso de escucha, deliberación y acción compartida con la sociedad:

- El programa Ekinez Ikasi que a través del proceso metodológico de Action Learning desarrolla diversos proyectos de escucha activa a la sociedad y también a colectivos internos de la Diputación Foral de Gipuzkoa.

- El proyecto de Presupuestos Participativos donde la ciudadanía plantea sus prioridades a la hora de configurar el presupuesto de la institución.

- La convocatoria de Proyectos de Ciudadanía en el que la ciudadanía plantea y desarrolla proyectos de innovación social.

- El programa Udal Etorkizuna Eraikiz en el que 12 ayuntamientos del territorio de Gipuzkoa están desarrollando en su municipio proyectos de Gobernanza Colaborativa tomando como referencia Etorkizuna Eraikiz.

- La Mesa de Partidos Políticos en el que participan todos los partidos que tienen representación en el parlamento territorial para deliberar sobre la agenda política del futuro y adoptar decisiones de manera compartida. 
Todos estos programas muestran una importante estructura de deliberación compartida entre la Diputación Foral de Gipuzkoa, los actores institucionales, políticos y sociales del territorio.

2) Gipuzkoa Lab: Es el espacio donde se desarrollan proyectos de experimentación activa con el objetivo de incorporar una nueva agenda política al desarrollo de las políticas públicas de la Diputación Foral de Gipuzkoa. Más de 40 proyectos experimentales están permitiendo experimentar sobre inteligencia artificial, participación de los/as trabajadores/as en la empresa, conciliación, exclusión social, diversidad, implicación comunitaria en el sistema de cuidados, etc. La agenda 2030, las misiones establecidas por parte de la Unión Europea o la estrategia RIS III de especialización inteligente del Gobierno Vasco materializado en el Plan de Ciencia y Tecnología constituyen los marcos de referencia para el establecimiento de la nueva agenda política y de los proyectos planteados.

Cada proyecto vincula a cuatro actores fundamentales: la sociedad civil (actores representativos en los respectivos sectores), la universidad (o actores vinculados a los ámbitos de generación de conocimiento), la administración pública (la Diputación Foral de Gipuzkoa, ayuntamientos, Gobierno Vasco, etc.) y actores con capacidad de producción o generación de conocimiento a nivel internacional (OCDE, Climate Kic, etc.). El proceso de experimentación se lleva a cabo a través del método de investigación-acción en el que destacan cuatro pasos fundamentales: el diseño compartido de los objetivos, el acuerdo sobre los contenidos y la metodología a desarrollar en el proceso de experimentación, el desarrollo de la investigación y la evaluación del proceso y los resultados. El objetivo de los proyectos de experimentación es aprender y extraer conclusiones en orden a incorporar mejoras en las políticas públicas.

Algunos de los proyectos desarrollados en el marco de este espacio son: Adinkide, Bizilagun Sarea, Centro de apoyo al talento deportivo, Chatbot, Compromiso Ciudadano por el clima, Conciliación e igualdad, ELKAR-EKIN lanean, La participación de los trabajadores en las empresas, Educación afectivo sexual, Emakumeen etxeak, Etxean Bizi, Gazteon Sarelan, Gipuzkoa CITYGML, Impulso de las mujeres en los Consejos de Administración de las empresas, entre otros.

El desarrollo de estos proyectos muestra que Gipuzkoa Lab se ha configurado como un espacio importante para construir relaciones de confianza entre diversos actores, un factor de aceleración y experimentación de contenidos avanzados y una fuente de aprendizaje para vincular los resultados de la experimentación con las políticas públicas.

3) Los Centros de Referencia de Etorkizuna Eraikiz: Los Centros de Referencia son espacios de trabajo público-privado-sociales para fortalecer determinados sectores de naturaleza estratégica para el futuro del territorio. La forma jurídica que adoptan estos espacios es la de una fundación, pero también la de un consorcio u otro tipo de institucionalización que se inserta en otras organizaciones más amplías a través de la fórmula de un convenio. Con independencia de la fórmula jurídica, los Centros de Referencia son formas de institucionalizar la Gobernanza Colaborativa.

El punto de partida de los Centros de Referencia se sitúa en la fortaleza de la sociedad gipuzkoana a la hora de abordar los retos estratégicos del territorio. El Gobierno Vasco a través de la estrategia de especialización inteligente RIS III, establece las líneas fundamentales por las que es necesario apostar para construir el futuro de Euskadi y de Gipuzkoa. La Diputación Foral de Gipuzkoa decide apostar, en coherencia con la estrategia RIS III, por el fortalecimiento de determinados sectores teniendo en cuenta las condiciones y potencialidades ya existentes en el territorio. En esta línea de trabajo se han creado los siguientes Centros de Referencia:

- MUBIL: El objetivo es que el territorio se convierta en un referente en cuanto a la nueva movilidad y la electrificación. Además de estar técnicamente preparados 
para ello, es necesario que a través del modelo Living Lab se consiga ser un territorio de demostración de nuevas investigaciones y desarrollos del sector. En este sentido, el territorio pretende estar preparado para aprovechar las nuevas actividades económicas que puedan surgir en torno a esta transformación, ofreciendo nuevas oportunidades de diversificación a diversas empresas que actualmente se dedican a la fabricación de piezas para el sector de la automoción.

- 2deo: El objetivo es incrementar la producción y consumo de los productos audiovisuales en euskera, promoviendo nuevos contextos de creatividad, experimentando en los modelos de producción y nuevos formatos, $\mathrm{y}$ multiplicando los canales de difusión. Todo ello, en colaboración con los agentes del sector audiovisual y la ciudadanía.

- Adinberri: El Centro de Referencia AdinBerri aspira a que las personas mayores tengan hábitos de vida saludables para prevenir y prolongar su autonomía, así como, disponer del apoyo preciso para mantener el control sobre su vida cotidiana y seguir viviendo una vida con sentido acorde a sus valores y preferencias en el marco de una sociedad para todas las generaciones, inclusiva y cohesionada. Adinberri tiene como objetivo desarrollar e impulsar una economía en torno al envejecimiento que dé respuesta al colectivo senior.

- Arantzazulab: Es un laboratorio de innovación social que tiene como objetivo ser un espacio de reflexión y experimentación innovadora sobre los retos del futuro de Gipuzkoa y de la sociedad vasca. El objetivo es ser un centro de innovación social referencial y un punto de encuentro de la ciudadanía para lograr una sociedad más justa, cohesionada, competitiva y humana.

- Elkar-Ekin Lanean: Es una estrategia para la mejora de la empleabilidad y la lucha contra la exclusión social. El objetivo es lograr una mejor orientación de las personas en la consecución de un empleo mejor. Y, al mismo tiempo, se persigue el fomento de políticas que acerquen un empleo digno a personas que se encuentren en riesgo de padecer exclusión social.

- LABE: Desde la perspectiva profesional, el objetivo es desarrollar una aceleradora de negocios de gastronomía digital, además de experimentar y ensayar la idea del restaurante 4.0. Desde la perspectiva ciudadana, el objetivo es impulsar nuevos sistemas de elaboración de alimentos y la gestión inteligente de productos y existencias.

- Naturklima: Está configurado como un centro multidisciplinario de carácter público para la generación de capacidad institucional, técnica y social. La misión del Centro de Referencia es hacer frente a los impactos del cambio climático, aportando conocimiento, valor y riqueza a la sociedad y al sector empresarial, convirtiéndose en acelerador de la transición socio-ecológica. Naturklima centralizará las políticas para combatir el calentamiento global y promoverá la economía circular, al tiempo que ayudará a Gipuzkoa a alinear su estrategia con el resto de centros de excelencia europeos.

- Ziur: Gipuzkoa es un territorio en el que se ubican un gran número de empresas especializadas y punteras en ciberseguridad, centros tecnológicos destacados, y universidades que ofrecen una importante cobertura académica en esta materia. El objetivo es impulsar un proyecto sólido, donde conocimiento y tecnología se pondrán al servicio de la competitividad industrial.

- Badalab: Es un consorcio público-social constituido por la Diputación Foral de Gipuzkoa, el Ayuntamiento de Rentería y organismos e instituciones sociales del euskera con la finalidad de impulsar el uso social del euskera. 


\subsection{El funcionamiento del modelo Etorkizuna Eraikiz}

La Figura 2 muestra con claridad el funcionamiento de Etorkizuna Eraikiz. Proiektuen Bulegoa es el órgano encargado de garantizar la conectividad y la lógica relacional entre Gipuzkoa Taldean (espacio de deliberación y proposición) y Gipuzkoa Lab (espacio de experimentación y aprendizaje). Analiza las propuestas de Proyectos Experimentales y decide, en su caso, su aprobación y puesta en marcha.

Figura 2. Funcionamiento del Modelo Etorkizuna Eraikiz.



Fuente: Diputación Foral de Gipuzkoa (2019).

El funcionamiento de la estrategia integra un núcleo estable de personas que son nombradas a propuesta del Diputado General de Gipuzkoa. Sus funciones e importancia radican en garantizar la conectividad entre Gipuzkoa Taldean y Gipuzkoa Lab. Hacer el seguimiento de la actividad desarrollada en ambos espacios. Valorar las propuestas y aprobar, en su caso, la puesta en marcha de los Think Tanks y de los proyectos experimentales. Elaborar la Memoria Anual de actividad de Etorkizuna Eraikiz e implementar y elaborar el Informe Bianual de Evaluación de este.

La aplicación de Etorkizuna Eraikiz se apoya en tres líneas transversales: Investigación, Internacionalización y Socialización que deben estar presentes de una manera activa como marcos de referencia y perspectivas compartidas de las prácticas de deliberación y experimentación que se desarrollan en los espacios existentes. Son fundamentales para producir información, conocimiento y aprendizaje que ayuden a construir enfoques y soluciones concretas a los retos presentes y futuros del territorio.

De otro lado, existen tres procesos de soporte: Gestión, Socialización, Comunicación que deben aportar permanentemente el servicio y el complemento que garanticen a las actividades desarrolladas en Gipuzkoa Taldean y Gipuzkoa Lab herramientas de apoyo (gestión), marcos para interactuar y compartir con personas y grupos (socialización) y un alcance y difusión más amplios (comunicación). 


\section{Conclusiones}

El modelo de Gobernanza Colaborativa en Gipuzkoa es producto de un proceso de reflexión y planificación estratégica llevado a cabo por la Diputación Foral de Gipuzkoa para la reorientación de las políticas públicas y la innovación del sistema de gobernanza institucional. La razón de ser de esta estrategia responde al reto que se ha autoimpuesto la Diputación Foral de Gipuzkoa de construir y desarrollar la agenda política de forma colaborativa, sumando conocimientos y experiencias que ayuden a construir el futuro de forma conjunta.

Con esta iniciativa la Diputación Foral de Gipuzkoa pretende buscar un nuevo equilibrio entre la política institucionalizada y la ciudadanía, entre la administración y la sociedad civil. Se trata de propiciar nuevas formas de relación, comunicación y cooperación con la ciudadanía.

El Programa Etorkizuna Eraikiz es, por lo tanto, una iniciativa promovida, guiada y financiada por los responsables políticos del gobierno de la Diputación Foral de Gipuzkoa y no de una agencia pública o una red intermedia. Etorkizuna Eraikiz es, de hecho, un ensayo para tratar de estabilizar un nuevo modelo de gobernanza en Gipuzkoa, estableciendo mecanismos ordinarios de escucha, deliberación y participación en los que agentes y agencias sociales del territorio participan para definir primero y desarrollar después la agenda pública.

El modelo de innovación política en Gipuzkoa propuesto por Etorkizuna Eraikiz no atiende solo al proceso sino también al resultado. Así, en términos de proceso, la intención explícita es la de implicar a un número cada vez mayor y más diverso de actores sociales en la deliberación y en la toma de decisiones políticas. Pero, en términos de resultados, el modelo persigue generar resultados políticos relevantes, efectivos y aplicables.

Un programa de escucha (deliberación) y práctica (experimentación), como es el caso de Etorkizuna Eraikiz, tiene necesariamente la vocación de crear espacios que promuevan el aprendizaje, el intercambio, la comunicación y la transferencia entre actores diferentes. Estos espacios deben ser propicios para promover dinámicas innovadoras para el abordaje y la resolución de los problemas públicos. Su papel es igualmente relevante en la canalización de la información, la comunicación y la facilitación de la relación entre los actores políticos e institucionales y la ciudadanía.

El desarrollo de un Programa de Gobernanza Colaborativa como Etorkizuna Eraikiz podrá ser más fructífero cuanto mayor sea el capital social que facilite la disposición y el comportamiento colaborativo de la ciudadanía. En este sentido, Gipuzkoa posee atributos favorecedores de las dinámicas colaborativas debido al capital social y a las estructuras comunitaristas presentes en el territorio.

Finalmente, vale la pena destacar que la capacidad de la política y de los gobiernos para alcanzar objetivos beneficiosos para la sociedad se apoya cada vez menos en sus propios recursos y fortalezas propias, y depende cada vez más de su capacidad para liderar colaboraciones y coaliciones con otros agentes sociales. Etorkizuna Eraikiz parte de la constatación anterior y promueve fórmulas de escucha y experimentación que necesitan el concurso y la cooperación de distintos agentes sociales.

\section{Referencias}

Aguilar Villanueva, L. (2008). Gobernanza: normalización conceptual y nuevas cuestiones. Cuadernos de Liderazgo, 17(1), 1-17.

Aguilar Villanueva, L. (2020). Democracia, gobernabilidad y gobernanza. Instituto Nacional Electoral.

Ansell, C., \& Gash, A. (2008). Collaborative Governance in theory and practice. Journal of Public Administration Research and Theory, 18(4), 543-571. https://doi.org/10.1093/jopart/mum032 
Arellano, D., Sánchez, J., \& Retana, B. (2014). ¿Una o varios tipos de Gobernanza? Cuadernos de Gobierno y Administración Pública, 1(2), 117-137. https://doi.org/10.5209/rev_CGAP.2014.v1.n2.47538

Atkinson, M., \& Coleman, W. (1992). Policy networks, policy communities and the problems of governance. Governance, 5(2), 154-180. https://doi.org/10.1111/j.1468-0491.1992.tb00034.x

Bauman, Z. (2017). La sociedad sitiada: Fondo de Cultura Económica de España.

Beck, U. (1998). ¿Qué es la globalización? Falacias del globalismo, respuestas a la globalización. Paidós.

Bourdieu P. (1983). The forms of capital. En J. Richardson (Ed.), Handbook of Theory and Research for the Sociology of Education (pp. 241-258). Greenwood Press.

Calame, P., \& Talmant, A. (1997). L'État au cour: le meccano de la gouvernance. Desclée de Brouwer.

Coleman, J. S. (1988). Social capital in the creation of human capital. American Journal of Sociology, 94, S95S120.

Coleman, J. S. (1990). Foundations of social theory. Harvard University Press.

Canto, R. (2012). Gobernanza y democracia: De vuelta al río turbio de la política. Gestión y Política Pública, 21(2), 333-374.

Castells, M. (2018). Ruptura. La crisis de la democracia liberal. Alianza.

Coghlan, D., \& Brannick, T. (2001). Doing Action Research in Your Own Organization. Sage.

Conejero, E. (2016). Innovación social y nuevos modelos de gobernanza para la provisión de bienes y servicios públicos. Estado, Gobierno y Gestión Pública, (27), 5-39.

Cooper, T. (2005). Civic engagement in the twenty-first century: Toward a scholarly and practical agenda. Public Administration Review, 65(5), 534-535. https://doi.org/10.1111/j.1540-6210.2005.00480.x

Cooper, T., Bryer, T., \& Meek, J. (2006). Citizen-centered collaborative public management. Public Administration Review, 66, 76-88. https://doi.org/10.1111/j.1540-6210.2006.00668.x

De Venanzi, A. (2002). Globalización y corporación: el orden social en el siglo XXI. Anthropos Editorial.

Diputación Foral de Gipuzkoa. (2019). Modelo Etorkizuna Eraikiz. Diputación Foral de Gipuzkoa.

Emerson, K., Nabatchi, T., \& Balogh, S. (2012). An integrative framework for collaborative governance. Journal of Public Administration Research and Theory, 22(1), 1-29. https://doi.org/10.1093/jopart/mur011

Longo, F. (2020). La administración pública en la era del cambio exponencial: Hacia una gobernanza exploratoria. Pertsonak eta Antolakunde Publikoak Kudeatzeko Euskal Aldizkaria= Revista Vasca de Gestión de Personas y Organizaciones Públicas, (3), 52-73.

Fukuyama, F. (1995). Trust: The Social Virtues and the Creation of Prosperity. Free Press.

Fung, A. (2006). Varieties of participation in complex governance. Public Administration Review, 66, 66-75. https://doi.org/10.1111/j.1540-6210.2006.00667.x

Giddens, A. (2007). Un mundo desbocado, los efectos de la globalización en nuestras vidas. Taurus.

Guilluy, C. (2019). No society: El fin de la clase media occidental. Taurus. 
Hardy, C., \& Phillips, N. (1998). Strategies of engagement: Lessons from the critical examination of collaboration and conflict in an interorganizational domain. Organization Science, 9(2), 217-230. https://doi.org/10.1287/orsc.9.2.217

Head, B. W. (2007). Community engagement: participation on whose terms? Australian Journal of Political Science, 42(3), 441-454. https://doi.org/10.1080/10361140701513570

Ilyin, M. (2013). Governance: What is behind the word? International Political Science Association IPSA, 37(1), 3-4.

Kenis, P., \& Schneider, V. (1991). Policy networks and policy analysis: scrutinizing a new analytical toolbox. En B. Marin \& R. Mayntz (Eds.), Policy networks: Empirical evidence and theoretical considerations (pp. 25-59). Campus Verlag.

Kooiman, J. (2000). Societal governance: Levels, models, and orders of social-political interaction. In J. Pierre (Ed.), Debating Governance (pp. 138-66). Oxford University Press.

López, J. \& Lavié, J.M. (2010). Liderazgo para sostener procesos de innovación en la escuela. Profesorado. Revista de Currículum y Formación de Profesorado, 14(1), 71-92.

Mair, P. (2017). Gobernando el vacío. La banalización de la democracia occidental. Alianza.

Mounk, Y. (2018). El pueblo contra la democracia. Paidós.

Natera Peral, A. (2005). Nuevas estructuras y redes de gobernanza. Revista Mexicana de Sociología, 67(4), 755791.

O'Leary, R., \& Bingham, L. (2009). The collaborative public manager: new ideas for the twenty-first century. Georgetown University Press.

Ospina, S. (2006). Gobernanza y liderazgos para el cambio social. Revista del CLAD Reforma y Democracia, (35), 1-17.

Peters, G., \& Pierre, J. (2005). ¿Por qué ahora el interés por la gobernanza? La gobernanza hoy, 10, 37-56.

Piketty, T. (2021). Una breve historia de la igualdad. Deusto.

Putnam, R. (1993). The prosperous community. The American Prospect, 4(13), 35-42.

Putnam, R. (2000). Bowling Alone: The Collapse and Revival of American Community. Simon \& Schuster.

Run, B., Huiting, Q., \& Oszlak, O. (2018). Gobernanza Colaborativa: las contingencias del poder compartido en Estado Abierto. Revista sobre el Estado, la Administración y las Políticas Públicas, 2(3), 47-90.

Rhodes, R. (1999). Governance and networks. En G. Stoker (Ed.), The New Management of British Local Governance (pp. xii-xxvi). Macmillan.

Rhodes, R. (2007). Understanding governance: Ten years on. Organization Studies, 28(8), 1243-1264. https://doi.org/10.1177/0170840607076586

Saffer, A. (2016). A message-focused measurement of the communication dimension of social capital: Revealing shared meaning in a network of relationships. Journal of Public Relations Research, 28(3-4), 170-192. https://doi.org/10.1080/1062726X.2016.1228065

Simone, R. (2016). El Hada democrática. Taurus. 
Sirianni, C. (2010). Investing in democracy: Engaging citizens in collaborative governance. Brookings Institution Press.

Smith, N. (2010). The public administrator as collaborative citizen: Three conceptions. Public Administration Quarterly, 34, 238-262.

Sørensen, E., \& Torfing, J. (2009). Making governance networks effective and democratic through metagovernance. Public Administration, 87(2), 234-258. https://doi.org/10.1111/j.1467-9299.2009.01753.x

Subirats, J. (2010). Si la respuesta es gobernanza, ¿cuál es la pregunta? Factores de cambio en la política y en las políticas. EKONOMIAZ. Revista vasca de Economía, 74(02), 16-35.

Whittingham Munévar, M.V. (2010). ¿Qué es la gobernanza y para qué sirve? Revista Análisis Internacional, (2), 219-236.

\section{(c) $($ () $\odot$}

(C) Attribution-NonCommercial-NoDerivatives 4.0 International (CC BY-NC-ND 4.0)

https://creativecommons.org/licenses/by-nc-nd/4.0/ 